\title{
Predictors of early postoperative hypocalcemia after total parathyroidectomy in renal hyperparathyroidism
}

\author{
Poh Guan Tan', Imi Sairi Ab. Hadi' ${ }^{2}$ Zalina Zahari ${ }^{3}$, Maya Mazuwin Yahya ${ }^{1,4}$, Wan Zainira Wan Zain ${ }^{1,4}$, \\ Michael Pak-Kai Wong ${ }^{1,4}$, Rosnelifaizur Ramely ${ }^{1,4}$, Mohd Nizam Md Hashim ${ }^{1,4}$, Syed Hassan Syed Abd. Aziz ${ }^{1,4}$, \\ Zaidi Zakaria ${ }^{1,4}$, Andee Dzulkarnaen Zakaria ${ }^{1,4}$ \\ ${ }^{1}$ Department of Surgery, School of Medical Sciences, Universiti Sains Malaysia (USM), Kubang Kerian, Kelantan, Malaysia \\ ${ }^{2}$ Department of Surgery, Hospital Raja Perempuan Zainab 2, Kota Bharu, Kelantan, Malaysia \\ ${ }^{3}$ Faculty of Pharmacy, Universiti Sultan Zainal Abidin (UniSZA), Besut Campus, Besut, Terengganu, Malaysia \\ ${ }^{4}$ Hospital Universiti Sains Malaysia, Kubang Kerian, Kelantan, Malaysia
}

\begin{abstract}
Purpose: Patients with secondary hyperparathyroidism are at high risk for developing postoperative hypocalcemia. However, there are limited data regarding predictors of postoperative hypocalcemia in renal failure patient with secondary hyperparathyroidism. This study aimed to determine the clinical presentations of renal hyperparathyroidism and the predictors of early postoperative hypocalcemia after total parathyroidectomy.

Methods: Data of patients with renal hyperparathyroidism who underwent total parathyroidectomy between January 2007 to December 2014 were reviewed retrospectively. Patients were divided into 2 cohort groups according to their serum calcium levels within 24 hours of parathyroidectomy: the hypocalcemia group (calcium levels of $2 \mathrm{mmol} / \mathrm{L}$ or less), and the normocalcemia group (calcium levels more than $2 \mathrm{mmol} / \mathrm{L}$ ). With the use of multivariable logistic regression analyses, the predictors of early postoperative hypocalcemia after total parathyroidectomy in patients with renal hyperparathyroidism were investigated.

Results: Among 68 patients, 56 patients (82.4\%) were symptomatic preoperatively. Fifty patients (73.5\%) presented with bone pain and 14 patients (20.6\%) had muscle weakness. Early postoperative hypocalcemia occurred in 25 patients (36.8\%). Preoperative alkaline phosphatase level was the predictor of early postoperative hypocalcemia ladjusted odds ratio, 1.004; 95\% confidence interval, 1.001-1.006; $P=0.002$ ).

Conclusion: Results from our study show that most of the patients with renal hyperparathyroidism were symptomatic preoperatively and the most common clinical presentations were bone pain and muscle weakness. The significant predictor of early postoperative hypocalcemia after total parathyroidectomy was the preoperative alkaline phosphatase levels.

[Ann Surg Treat Res 2020;98(1):1-6]
\end{abstract}

Key Words: Hypocalcemia, Parathyroidectomy, Parathyroid hormone, Renal insufficiency, Secondary hyperparathyroidism

\section{INTRODUCTION}

Hyperparathyroidism is a disease characterized by inappropriately elevated parathyroid hormone (PTH) levels. Prevalence for hyperparathyroidism is around $0.3 \%$ in the general population [1]. Primary hyperparathyroidism is a condition characterized by inappropriate excess secretion of PTH leading to hypercalcemia and hypophosphatemia. The majority of primary
Received June 12, 2019, Revised September 10, 2019,

Accepted October 23, 2019

Corresponding Author: Zalina Zahari

Faculty of Pharmacy, Universiti Sultan Zainal Abidin (UniSZA), Besut

Campus, 22200 Besut, Terengganu, Malaysia

Tel: +609-6993119, Fax: +609-6993004

E-mail: zalinazahari@unisza.edu.my

ORCID: https://orcid.org/0000-0003-1459-8958
Copyright (c) 2020, the Korean Surgical Society

(c) Annals of Surgical Treatment and Research is an Open Access Journal. All articles are distributed under the terms of the Creative Commons Attribution NonCommercial License (http://creativecommons.org/licenses/by-nc/4.0/) which permits unrestricted non-commercial use, distribution, and reproduction in any medium, provided the original work is properly cited. 
hyperparathyroidism is caused by autonomous PTH secretion from a solitary parathyroid adenoma.

Secondary hyperparathyroidism (SHPT) refers to excessive secretion of PTH in response to hypocalcemia and associated hyperplasia of the parathyroid glands. SHPT enhances vascular calcification with increased cardiovascular mortality. Chronic renal failure, hypermagnesemia, osteoporosis and osteomalacia/rickets can cause SHPT. By far, the most common cause of SHPT is a chronic renal failure, though its clinical effects were not well elucidated until recently. Pathogenesis of SHPT is complicated, every aspect of renal failure from decreased synthesis function to metabolic abnormalities to even treatment of renal failure contribute to it [2]. Factors that lead to hypertrophy and hyperplasia transformation of the parathyroid gland with subsequent elevation of PTH in order to normalize serum calcium level such as hyperphosphataemia and hypocalcemia, reduce renal 1-alpha-hydroxylase and calcitriol, bony resistance to PTH (if excessive PTH), change in PTH set point (rise in set point), and aluminium intoxication.

Initial treatment for SHPT is aimed to bring the serum calcium and phosphate to a physiological level to prevent overstimulation of parathyroid glands and hence overproduction of PTH. Medical therapy includes calcium supplement, vitamin D supplement, phosphate-poor diets, aluminum-binding agents, hemodialysis with calcium-enriched dialysate and calcimimetic agent, Cinacalcet [2]. Despite the advent of new therapeutic agents, the rate of parathyroidectomy did not change significantly over the past 20 years. Parathyroidectomy is indicated in patients who failed medical treatment [3,4], patients with intractable bone pain/pruritus, fracture, and patients with symptomatic ectopic calcification [5].

Hypocalcemia or even hungry bone syndrome is a wellknown and severe complication after parathyroidectomy [68], but not all patient develop this complication. Thus, it would be beneficial and clinically significant to identify the high-risk patient group that develops postoperative hypocalcemia/hungry bone syndrome in order to monitor them more closely during the postoperative period. This study was designed to determine the clinical presentations of renal hyperparathyroidism and the predictors of early postoperative hypocalcemia after total parathyroidectomy.

\section{METHODS}

\section{Study design and setting}

This is a retrospective review of patients who underwent total parathyroidectomy for SHPT over 8 years from January 2007 to December 2014 in Hospital Raja Perempuan Zainab II (HRPZ II), Malaysia.

\section{Ethical approval}

The Universiti Sains Malaysia (USM) and Ministry of Health (MOH), Malaysia Institutional Review Board (approval number: USM/JEPeM/16020065 and NMRR-16-250-29616) approved this study.

\section{Participants}

There were 88 patients with SHPT who underwent parathyroidectomy retrieved from the records of the operation theater in HRPZ II from January 1st, 2007 to December 31st, 2014. Sixtyeight patients met the eligibility criteria.

The inclusion criteria for this study included renal failure patients with SHPT who underwent total parathyroidectomy during the study period. Patients with renal hyperparathyroidism who underwent parathyroidectomy with incomplete data or missing data and aged below 16 years old were excluded.

\section{Sample size estimation}

The sample size was calculated using 2 means and 2 proportions formula for both continuous and categorical variables, respectively, to obtain the appropriate sample size for the predictors of early postoperative hypocalcemia after total parathyroidectomy in renal failure patients with SHPT. The calculation was done using the PS: Power and Sample Size Calculation Version 3.0.12 (DalePlummer, Nashvile, TN, USA). The final targeted sample size was determined by considering a $20 \%$ drop-out rate. The estimated sample size for this study was 56 samples.

\section{Data collection}

This study involved secondary data collection. The list of patients with SHPT who underwent parathyroidectomy in HRPZ II during the 8 years from the 1st of January 2007 until the 31st of December 2014 was obtained from the records of the operation theater.

In HRPZ II, initial bilateral neck exploration and total parathyroidectomy without autotransplantation were performed for all patients who required surgical intervention since the renal transplantation rate is low in Malaysia and the majority of patients require long-term hemodialysis after parathyroidectomy, and consequently the risk of recurrence is high and not negligible.

Patients' medical records were reviewed in detail to obtain the clinical data and biochemical parameters. All information needed is recorded in the data collection form to reduce bias. Variables that were required for the study were age, gender, duration of dialysis, main complaint, occurrence of renal hyperparathyroidism, preoperative blood investigation (serum calcium, albumin, phosphate, ALP, intact PTH [iPTH], and creatinine levels), postoperative calcium levels (within 24 hours of total parathyroidectomy before calcium infusion was started), 
and glands histopathological results.

Patients were divided into 2 groups based on the postoperative calcium levels within 24 hours: normocalcemia (calcium levels more than $2 \mathrm{mmol} / \mathrm{L}$ ) and hypocalcemia (calcium levels $2 \mathrm{mmol} / \mathrm{L}$ or $<2 \mathrm{mmol} / \mathrm{L}$ ) group [6,9].

\section{Statistical analysis}

The data were analysed by univariable analysis (simple logistic regression) and multivariable analysis (multiple logistic regression). Early postoperative hypocalcemia after total parathyroidectomy was the outcome variable. Independent variables were possible predictors of early postoperative hypocalcemia after total parathyroidectomy included age, sex, clinical presentation (symptomatic or asymptomatic), duration of hemodialysis, preoperative levels of calcium, albumin, phosphate, ALP, iPTH, and creatinine. In univariable analysis, the aim was to screen for important independent variables with a P-value less than $0.25(\mathrm{P}<0.25)$ and clinically significant variables [10]. multivariable analysis was used to run all variables from univariable analysis at one time.

In this study, several variables with $\mathrm{P}<0.25$ or clinically important such as age, preoperative levels of calcium, phosphate, ALP, iPTH, and creatinine were selected as continuous variables for multiple logistic regression based on the principle of parsimony, the best fit, biologically plausible, and statistically significant. Statistical analysis was carried out using IBM SPSS Statistics ver. 22.0 (IBM Co., Armonk, NY, USA). The limit of significance was set at 0.05 .

\section{RESULTS}

\section{Patient demographics and clinical profiles}

Among 88 patients with SHPT who underwent parathyroidectomy reviewed from the medical records, 68 patients met the inclusion and exclusion criteria and were included in the study. Twenty patients were excluded due to surgery for primary hyperparathyroidism $(n=10)$, incomplete data or missing data $(n=6)$, re-exploration after primary surgery $(n=$ 3), and aged less than 16 years old $(n=1)$.

Table 1 shows the characteristics of patients with renal hyperparathyroidism. In total, 47 males and 21 females were included in the study with an average age of 42.9 years old (standard deviation, 12.21). Among the 68 patients, 56 patients (82.4\%) were symptomatic preoperatively. Patients presented with different symptoms or a combination of symptoms. Fifty patients $(73.5 \%)$ presented with bone pain and 14 patients (20.6\%) had muscle weakness. Early postoperative hypocalcemia occurred in 25 patients (36.8\%).

\section{Predictors of early postoperative hypocalcemia after total parathyroidectomy in renal hyperparathyroidism}

Table 2 shows univariable analyses on the predictors of early postoperative hypocalcemia after total parathyroidectomy in patients with renal hyperparathyroidism. Using simple logistic regression, age (crude odds ratio [OR], 0.957; 95\% confidence interval [CI], 0.916-0.999; $\mathrm{P}=0.043$ ) and preoperative levels of ALP (crude OR, 1.004; 95\% CI, 1.001-1.006; P = 0.002) were seen to be significant with a P-value less than 0.05 . Simple logistic regression analyses showed that patients in the hypocalcemia

Table 1. Characteristics of patients with renal hyperparathyroidism $(\mathrm{n}=68)$

\begin{tabular}{|c|c|}
\hline Variable & Value \\
\hline Age $(y r)$ & $42.9(19-67)$ \\
\hline \multicolumn{2}{|l|}{ Sex } \\
\hline Male & $47(69.1)$ \\
\hline Female & $21(30.9)$ \\
\hline \multicolumn{2}{|l|}{ Race } \\
\hline Malay & $66(97.1)$ \\
\hline Chinese & $2(2.9)$ \\
\hline \multicolumn{2}{|l|}{ Preoperative clinical presentation } \\
\hline Asymptomatic & $12(17.6)$ \\
\hline Symptomatic & $56(82.4)$ \\
\hline \multicolumn{2}{|l|}{ Preoperative symptom ${ }^{a}$} \\
\hline Bone pain & $50(73.5)$ \\
\hline Muscle weakness & $14(20.6)$ \\
\hline Paraesthesia & $9(13.2)$ \\
\hline Headache/dizziness & $5(7.4)$ \\
\hline Renal stone & $5(7.4)$ \\
\hline Nausea and vomiting & $4(5.9)$ \\
\hline Constipation & $1(1.5)$ \\
\hline Peptic ulcer & $1(1.5)$ \\
\hline Psychosis/dementia & $1(1.5)$ \\
\hline Duration of renal failure (yr) & $7.16(2-17)$ \\
\hline Duration of hemodialysis (yr) & $7.27(2-15)$ \\
\hline \multicolumn{2}{|l|}{ Preoperative serum level } \\
\hline Calcium (mmol/L) & $2.52(1.56-3.5)$ \\
\hline Albumin $(g / L)$ & $37.81(26-49)$ \\
\hline Phosphate $(\mathrm{mmol} / \mathrm{L})$ & $1.95(0.75-3.04)$ \\
\hline Alkaline phosphatase (IU/L) & $401.41(65-2,578)$ \\
\hline Intact parathyroid hormone $(\mathrm{pg} / \mathrm{mL})$ & $615(77-2,000)$ \\
\hline Creatinine $(\mu \mathrm{mol} / \mathrm{L})$ & $815(133-1,444)$ \\
\hline \multicolumn{2}{|l|}{ Postoperative serum calcium level ${ }^{\text {b) }}$} \\
\hline Hypocalcemia & $25(36.8)$ \\
\hline Normocalcemia & $43(63.2)$ \\
\hline
\end{tabular}

Values are presented as mean (range) or number (\%).

Patients were divided into 2 groups based on the postoperative calcium levels within 24 hours: normocalcemia (calcium levels more than $2 \mathrm{mmol} / \mathrm{L}$ ) and hypocalcemia (calcium levels $2 \mathrm{mmol} /$ $\mathrm{L}$ or $<2 \mathrm{mmol} / \mathrm{L}$ ) group.

${ }^{a}$ Some patients had more than one preoperative symptom. ${ }^{\text {b) }}$ Postoperative serum calcium level within 24 hours of total parathyroidectomy before calcium infusion was started. 
Table 2. Predictors of early postoperative hypocalcemia after total parathyroidectomy in patients with renal hyperparathyroidism from simple logistic regression analyses $(n=68)$

\begin{tabular}{|c|c|c|}
\hline Variable & Crude OR $(95 \% \mathrm{Cl})$ & P-value \\
\hline $\operatorname{Age}^{\mathrm{a})}$ & 0.957 (0.916-0.999) & 0.043 \\
\hline \multicolumn{3}{|l|}{ Sex } \\
\hline Female & 1.000 & 0.879 \\
\hline Male & $1.086(0.375-3.144)$ & \\
\hline \multicolumn{3}{|l|}{ Clinical presentation } \\
\hline Asymptomatic & 1.000 & \\
\hline Symptomatic & $1.941(0.473-7.970)$ & 0.357 \\
\hline Duration of hemodialysis & $0.999(0.847-1.178)$ & 0.988 \\
\hline \multicolumn{3}{|l|}{ Preoperative serum level } \\
\hline Calcium ${ }^{\text {a) }}$ & $1.513(0.353-6.465)$ & 0.576 \\
\hline Albumin & $1.040(0.937-1.154)$ & 0.466 \\
\hline Phosphate & $2.194(0.812-5.930)$ & 0.121 \\
\hline Alkaline phosphatase $\mathrm{e}^{\mathrm{a})}$ & $1.004(1.001-1.006)$ & 0.002 \\
\hline Intact parathyroid hormone ${ }^{a)}$ & $1.001(1.000-1.002)$ & 0.121 \\
\hline Creatinine $^{\text {a) }}$ & $1.001(0.999-1.003)$ & 0.246 \\
\hline
\end{tabular}

$\mathrm{OR}$, odds ratio; $\mathrm{Cl}$, confidence interval.

a) Variables included in variable selection regression analysis.

group were significantly younger than normocalcemia group (38.9 years vs. 45.2 years). Preoperative level of ALP was significantly higher in hypocalcemia group (603 IU/L vs. 283 IU/ L).

In this dataset, age, preoperative levels of calcium, phosphate, ALP, iPTH, and creatinine were included as continuous variables in the multiple logistic regression based on 4 principles of the best fit, parsimonious, statistically significant, and clinically meaningful.

Multiple logistic regression showed that the preoperative ALP level was the predictor of early postoperative hypocalcemia after total parathyroidectomy in patients with renal hyperparathyroidism. After adjustment for age, preoperative levels of calcium, phosphate, iPTH, and creatinine, the results showed that higher preoperative ALP level resulted in greater odds of getting postoperative hypocalcemia (adjusted OR, 1.004; 95\% CI, $1.001-1.006 ; \mathrm{P}=0.002$ ).

\section{DISCUSSION}

The present study explored the clinical presentations of renal hyperparathyroidism and the predictors of early postoperative hypocalcemia after total parathyroidectomy. The clinical effects of renal hyperparathyroidism include refractory pruritus, bone or joints pain, muscular weakness, progressive soft tissue calcification, renal stone, constipation, peptic ulcer disease, spontaneous long bone fracture and even psychosis or dementia [9]. From this study, bone pain was observed as the most common clinical presentation followed by muscular weakness, paraesthesia, headache/dizziness, renal stone, nausea and vomiting, peptic ulcer, and psychosis/dementia. None of the patients suffered from a spontaneous long bone fracture.

Postoperative hypocalcemia is a well-known and severe complication after parathyroidectomy $[7,8,11]$. The incidence of postoperative hypocalcemia among patients with primary hyperparathyroidism who underwent parathyroidectomy has been reported to be $10 \%$ to $46 \%$, and among patient with SHPT has been reported to be up to $26 \%$ to $95 \%[6,7]$. This study has established that the proportion of postoperative hypocalcemia among the study subjects was $36.8 \%$, and the results are comparable to other published studies $[6,7]$. The pathogenesis of postoperative hypocalcemia is multifactorial and its primary mechanism is possible due to abrupt removal of PTH stimulation after parathyroidectomy; hence, excess osteoclastic activity stops, but osteoblastic activity continues causing excess calcium and phosphate to pass into bones [12].

Many prior studies have evaluated the predictors of postoperative hypocalcemia in patients with primary hyperparathyroidism; however, the study of predictors of postoperative hypocalcemia in SHPT in renal failure patients is still limited [12-15]. Few studies have attempted to identify predictors for early postoperative hypocalcemia in SHPT with mixed results. Previous studies have identified several predictors for the occurrence of early postoperative hypocalcemia after parathyroidectomy, which included the age of patients, preoperative levels of calcium, alkaline phosphatase, and albumin and weight of the parathyroid gland $[2,6,9,16]$. A study by Hamouda et al. [6] involved 76 patients in Saudi Arabia aimed to identify the risk factors for developing early postoperative hypocalcemia after parathyroidectomy for SHPT has concluded that age, level of preoperative calcium, ALP, and serum albumin correlated with early postoperative hypocalcemia.

ALP level elevates due to increased osteoblastic activity in hyperparathyroidism patients secondary to stimulation from the high PTH level and is related to the degree of bone disease $[17,18]$. Patients with higher preoperative ALP are more prone to develop postoperative hypocalcemia or even hungry bone syndrome $[2,6,9,19]$. A regional study involving 2 tertiary institutions (Tan Tock Seng Hospital Singapore and Kuala Lumpur General Hospital) reported a strong relationship between ALP and postoperative hypocalcemia in patients with primary hyperparathyroidism [13]. The study concluded that patients with preoperative ALP less than $340 \mathrm{IU} / \mathrm{L}$ are unlikely to develop symptomatic postoperative hypocalcemia (100\% sensitivity, $95 \%$ specificity) and also recommended preoperative ALP to be used to complement clinical protocol for postoperative hypocalcemia management in parathyroidectomy patients [13].

Patient with SHPT develops more profound postoperative hypocalcemia. As such, preoperative predictors of hypocalcemia are particularly crucial for postoperative management [9]. 
The present study revealed that patients in the hypocalcemia group presented with a higher mean of preoperative ALP level compared with the normocalcemia group (603 IU/L vs. $283 \mathrm{IU} /$ L, respectively). Using multiple logistic regression analysis, high preoperative ALP exhibited positive predictive values of development of early postoperative hypocalcemia with an adjusted odds ratio of 1.004. Patients with high preoperative ALP are highly recommended to be monitored more closely in the early postoperative period, preferably in the high dependency unit, along with more frequent serum calcium monitoring to avoid the complication of early postoperative hypocalcemia. Postoperative empirical intravenous calcium supplementation should be given in the immediate postoperative period and at a higher dose for the high-risk group. When oral intake is feasible, oral vitamin D and calcium supplement should be started [20]. By implementing this protocol, severe hypocalcemia can be avoided and an uneventful postoperative period can be secured.

Age had been identified as one of the predictors for postoperative hypocalcemia in patients with primary hyperparathyroidism going back to the year 1988 [21]. Advanced age was associated with postoperative hypocalcemia in primary hyperparathyroidism and a few recent studies suggested that elderly patients usually have vitamin D deficiency and more inadequate oral intake: therefore, aging was associated with an increased risk of postoperative hypocalcemia [19,22]. In contrast, studies by Torer et al. [9] and Hamouda et al. [6] involving only SHPT patients found that younger age group patients are more prone to develop postoperative hypocalcemia after total parathyroidectomy. The present study on patients with SHPT, however, showed no association between age and postoperative hypocalcemia. The mean age for hypocalcemia group was lower (38.9 years vs. 45.2 years) and univariable analysis showed a significant association but not significant in subsequent multiple logistic regression analysis.

Preoperative PTH level is related to the severity of renal osteodystrophy. Therefore, a positive association between the increased level of preoperative serum PTH with postoperative hypocalcemia is expected. Patients in hypocalcemia group showed a higher level of the preoperative PTH than the normocalcemia group $(729.51 \mathrm{pg} / \mathrm{mL}$ vs. $548.50 \mathrm{pg} / \mathrm{mL})$. However, the difference was not statistically significant. Similarly, some studies also found that there was no association between preoperative PTH and early postoperative hypocalcemia

\section{[6,7,9,23-25].}

Ellul et al. [23] conducted a study to analyze the predictive value of preoperative serum phosphate level as an indicator for developing postoperative hypocalcemia and found that there was no significant association between the preoperative phosphate level and postoperative hypocalcemia following total parathyroidectomy. The difference was not significant on multivariable analysis in the present study.

None of the other factors evaluated including gender, duration of hemodialysis, duration of renal failure, serum calcium, serum albumin, and creatinine levels were predictive of the development of postoperative hypocalcemia in the renal patients with SHPT after total parathyroidectomy.

There were some limitations found in this study. This study carries the usual limitation of a retrospective study. Six patients' medical records were not found. Some documentation of the required information was weak and lacked complete data for 6 patients are potential confounding factors for this study. We advocate a more detailed prospective study regarding this matter in the future by obtaining more profound evidence. This would provide an evidence-based result and guidance in the management of patients with SHPT. More factors should be included in any future study to reduce the confounder effect. For example, preoperative urea level, serum vitamin D level and weight of parathyroid glands. Postoperative serum PTH should also be taken to exclude evidence of persistent disease after surgery. Postoperative serum PTH levels may further clarify the cause of hypocalcemia.

In conclusion, most of the patients with renal hyperparathyroidism were symptomatic preoperatively and the most common clinical presentations were bone pain and muscle weakness. The significant predictor of early postoperative hypocalcemia after total parathyroidectomy in renal failure patients with SHPT was the preoperative alkaline phosphatase levels. Patients with high preoperative alkaline phosphatase should be monitored more closely in the early postoperative period.

\section{CONFLICTS OF INTEREST}

No potential conflict of interest relevant to this article was reported.

\section{REFERENCES}

1. Lennard T. Endocrine surgery: a companion to specialist surgical practice. Ann
R Coll Surg Engl 2010;92:717.

2. Rashed A, Fahmi M, ElSayed M, Aboud
O, Asim M. Effectiveness of surgical parathyroidectomy for secondary hyperpara- 
thyroidism in renal dialysis patients in Qatar. Transplant Proc 2004:36:1815-7.

3. Gourgiotis S, Moustafellos P, Stratopoulos C, Vougas V, Drakopoulos S, Hadjiyannakis E. Total parathyroidectomy with autotransplantation in patients with renal hyperparathyroidism: indications and surgical approach. Hormones (Athens) 2006;5:270-5.

4. Saliba W, El-Haddad B. Secondary hyperparathyroidism: pathophysiology and treatment. J Am Board Fam Med 2009;22: 574-81.

5. Tominaga Y. Surgical treatment of secondary hyperparathyroidism due to chronic kidney disease. Ups J Med Sci 2006;111: 277-92.

6. Hamouda M, Dhia NB, Aloui S, El Mhamedi S, Skhiri H, Elmay M. Predictors of early post-operative hypocalcemia after parathyroidectomy for secondary hyperparathyroidism. Saudi J Kidney Dis Transpl 2013;24:1165-9.

7. Mittendorf EA, Merlino JI, McHenry CR. Post-parathyroidectomy hypocalcemia: incidence, risk factors, and management. Am Surg 2004;70:114-9.

8. Stewart ZA, Blackford A, Somervell H, Friedman K, Garrett-Mayer E, Dackiw AP, et al. 25-hydroxyvitamin $\mathrm{D}$ deficiency is a risk factor for symptoms of postoperative hypocalcemia and secondary hyperparathyroidism after minimally invasive parathyroidectomy. Surgery 2005;138:1018-25.

9. Torer N, Torun D, Torer N, Micozkadioglu H, Noyan T, Ozdemir FN, et al. Predictors of early postoperative hypocalcemia in hemodialysis patients with secondary hyperparathyroidism. Transplant Proc 2009;41:3642-6.

10. Hosmer DW, Lemeshow S, Sturdivant RX. Applied logistic regression. 3rd ed.
Hoboken: John Wiley \& Sons; 2013.

11. Kim WY, Lee JB, Kim HY, Woo SU, Son GS, Bae JW. Achievement of the National Kidney Foundation Kidney Disease Outcomes Quality Initiative: recommended serum calcium, phosphate and parathyroid hormone values with parathyroidectomy in patients with secondary hyperparathyroidism. J Korean Surg Soc 2013; 85:25-9.

12. Kaya C, Tam AA, Dirikoc A, Kilicyazgan A, Kilic M, Turkolmez S, et al. Hypocalcemia development in patients operated for primary hyperparathyroidism: can it be predicted preoperatively? Arch Endocrinol Metab 2016;60:465-71.

13. Loke SC, Tan AW, Dalan R, Leow MK. Preoperative serum alkaline phosphatase as a predictor for hypocalcemia post-parathyroid adenectomy. Int J Med Sci 2012;9: 611-6.

14. Zamboni WA, Folse R. Adenoma weight: a predictor of transient hypocalcemia after parathyroidectomy. Am J Surg 1986;152: 611-5.

15. Akerstrom G, Malmaeus J, Bergstrom R. Surgical anatomy of human parathyroid glands. Surgery 1984:95:14-21.

16. Tsai WC, Peng YS, Chiu YL, Wu HY, Pai MF, Hsu SP, et al. Risk factors for severe hypocalcemia after parathyroidectomy in prevalent dialysis patients with secondary hyperparathyroidism. Int Urol Nephrol 2015:47:1203-7.

17. Puccini M, Carpi A, Cupisti A, Caprioli R, Iacconi $\mathrm{P}$, Barsotti $\mathrm{M}$, et al. Total parathyroidectomy without autotransplantation for the treatment of secondary hyperparathyroidism associated with chronic kidney disease: clinical and laboratory longterm follow-up. Biomed Pharmacother 2010;64:359-62.
18. Stracke S, Keller F, Steinbach G, HenneBruns D, Wuerl P. Long-term outcome after total parathyroidectomy for the management of secondary hyperparathyroidism. Nephron Clin Pract 2009;111:c102-9.

19. Farese $S$. The hungry bone syndrome--an update. Ther Umsch 2007;64:277-80.

20. Loke SC, Kanesvaran R, Yahya R, Fisal L, Wong TW, Loong YY. Efficacy of an intravenous calcium gluconate infusion in controlling serum calcium after parathyroidectomy for secondary hyperparathyroidism. Ann Acad Med Singapore 2009; 38:1074-80.

21. Brasier AR, Nussbaum SR. Hungry bone syndrome: clinical and biochemical predictors of its occurrence after parathyroid surgery. Am J Med 1988;84:654-60.

22. Erbil Y, Bozbora A, Ozbey N, Issever H, Aral F, Ozarmagan S, et al. Predictive value of age and serum parathormone and vitamin d3 levels for postoperative hypocalcemia after total thyroidectomy for nontoxic multinodular goiter. Arch Surg 2007;142:1182-7.

23. Ellul D, Townsley RB, Clark LJ. Does the pre-operative serum phosphate level predict early hypocalcaemia following parathyroidectomy for primary hyperparathyroidism? Surgeon 2013;11:125-9.

24. Vasher M, Goodman A, Politz D, Norman J. Postoperative calcium requirements in 6,000 patients undergoing outpatient parathyroidectomy: easily avoiding symptomatic hypocalcemia. J Am Coll Surg 2010;211:49-54.

25. Randhawa PS, Mace AD, Nouraei SA, Stearns MP. Primary hyperparathyroidism: do perioperative biochemical variables correlate with parathyroid adenoma weight or volume? Clin Otolaryngol 2007; 32:179-84. 